\title{
Lattice calculation of the Polyakov loop and Polyakov loop correlators
}

\author{
Johannes Heinrich Weber ${ }^{1, a}$ (on behalf of TUMQCD collaboration) \\ ${ }^{1}$ Technische Universität München, Physik Department T30f, James-Franck-Str. 1, 85748 Garching, Ger- \\ many
}

\begin{abstract}
We discuss calculations of the Polyakov loop and of Polyakov loop correlators using lattice gauge theory. We simulate QCD with $2+1$ flavors and almost physical quark masses using the highly improved staggered quark action (HISQ). We demonstrate that the entropy derived from the Polyakov loop is a good probe of color screening. In particular, it allows for scheme independent and quantitative conclusions about the deconfinement aspects of the crossover and for a rigorous study of the onset of weak-coupling behavior at high temperatures. We examine the correlators for small and large separations and identify vacuum-like and screening regimes in the thermal medium. We demonstrate that gauge-independent screening properties can be obtained even from gauge-fixed singlet correlators and that we can pin down the asymptotic regime.
\end{abstract}

\section{Introduction}

QCD matter has a deconfined phase at high temperatures as well as a confined phase at low temperatures. In the confined phase, quarks and gluons are confined into hadrons and the scalar quark condensate indicates that chiral symmetries are broken spontaneously. In the deconfined phase the vanishing condensate indicates chiral symmetry restoration.

In pure Yang-Mills theory without sea quarks the Polyakov loop plays the role of an order parameter of the deconfinement phase transition, which is associated with the breaking of the gauge group's center symmetry. The logarithm of the Polyakov loop corresponds to the negative free energy of a static quark in temperature units [1]. As the Polyakov loop is not invariant under elements of the group's center, its expectation value vanishes exactly in the confined phase, i.e. the free energy of a static quark is infinite. Due to the onset of color screening at the phase transition, the expectation value of the Polyakov loop has a discontinuity and jumps to a finite value at the transition temperature, while its fluctuations diverge. Thus, the deconfinement transition is closely related to the onset of color screening [1].

The presence of sea quarks in full QCD breaks the center symmetry explicitly. The flux tubes rupture when a quark-antiquark pair is created from the sea and a pair of mesons is formed. Thus, the finite expectation value of the Polyakov loop in the confined phase of QCD, which is real and positive, is related to the energy of a static-light meson. The remnant of the phase transition of pure gauge theory

ae-mail: johannes.weber@tum.de 
is a smooth crossover transition in full QCD. The crossover temperature is usually determined from chiral susceptibilities, which can be defined as renormalization invariant quantities, cf. Ref. [2] and references therein. As the bare Polyakov loop is divergent, its necessary renormalization introduces a scheme dependence for its expectation value and for its susceptibility. If a deconfinement temperature obtained from the Polyakov loop is scheme dependent, it cannot be a fundamental property of QCD. Therefore, deconfinement in QCD is often discussed in terms of fluctuations of conserved charges, cf. [3] and references therein. Various observables in combination with different lattice actions yield somewhat different results for the crossover, leading to a long-standing confusion about its properties, cf. Ref. [4] and references therein.

In this contribution we discuss the deconfinement transition and color screening in QCD using the renormalized Polyakov loop or equivalently the free energy $F_{Q}$ of a static quark. We also consider the correlator of two Polyakov loops and its color singlet component and obtain the color-averaged free energy $F_{Q \bar{Q}}$ and the singlet free energy $F_{S}$ of a static quark anti-quark pair in full QCD.

\section{Lattice setup: Ensembles, observables and renormalization}

We study the Polyakov loop itself, the Polyakov loop correlator, the singlet Wilson line correlator and the cyclic Wilson loop in full QCD with $2+1$ flavors of sea quarks for a large range of temperatures and five different temporal extents $N_{\tau}=\{12,10,8,6,4\}$ of the lattice. The temperature $T$ is related to the gauge coupling $\beta=10 / g^{2}$ through the lattice spacing $a(\beta)$ and is defined as $T\left(\beta, N_{\tau}\right)=1 /\left(a(\beta) N_{\tau}\right)$. Taking the continuum limit at fixed temperature requires simultaneous variation of both $\beta$ and $N_{\tau}$, which is why numerical studies of QCD at finite temperature are quite demanding. We use lattices with aspect ratio $N_{\sigma} / N_{\tau}=4$, where $N_{\sigma}$ is the extent of each spatial direction. Furthermore we include two ensembles with aspect ratio $N_{\sigma} / N_{\tau}=6$ to control the volume dependence. We have physical strange quark mass and nearly physical light quark masses, $m_{l}=m_{s} / 20$, corresponding to a pion mass of $m_{\pi}=161 \mathrm{MeV}$ in the continuum limit. At high temperatures, where the quark mass dependence is mild, we also include some ensembles with $m_{l}=m_{s} / 5$. The gauge ensembles were generated with a tree-level improved Symanzik gauge action and highly improved staggered quarks (HISQ) by the HotQCD collaboration [2,5] and by the TUMQCD collaboration [6, 7]. We use the publicly available MILC code [8] in combination with libraries provided by the USCQD consortium [9].

We study a single static quark in terms of the Polyakov loop $P$. On the lattice, $P$ is defined as a normalized trace of a temporal Wilson line $W$ wrapping around the time direction once,

$$
P\left(\beta, N_{\tau}, \boldsymbol{x}\right)=\frac{1}{3} \operatorname{Tr} W\left(\beta, N_{\tau}, \boldsymbol{x}\right), \quad W\left(\beta, N_{\tau}, \boldsymbol{x}\right)=\prod_{\tau=1}^{N_{\tau}} U_{0}(\tau, \boldsymbol{x}),
$$

where $U_{0}(x=(\boldsymbol{x}, \tau))$ are the temporal link variables. Due to the trace, $P$ is gauge invariant. We denote the expectation value of the bare Polyakov loop as $L^{\text {bare }}\left(\beta, N_{\tau}\right)=\left\langle P\left(\beta, N_{\tau}, \boldsymbol{x}\right)\right\rangle$ and average it over the spatial lattice volume. We also study correlations of a static $Q \bar{Q}$ pair in terms of the Polyakov loop correlator $C_{P}$, the Wilson line correlator $C_{S}$ and the cyclic Wilson loop $W_{S}$. All three bare correlators are defined as ensemble averages (spatial average over $\boldsymbol{x}$ included) and read

$$
\begin{aligned}
C_{P}^{\text {bare }}\left(\beta, N_{\tau}, r\right) & =\left\langle P\left(\beta, N_{\tau}, \boldsymbol{x}\right) P^{\dagger}\left(\beta, N_{\tau}, \boldsymbol{x}+\mathbf{r}\right)\right\rangle, \\
C_{S}^{\text {bare }}\left(\beta, N_{\tau}, r\right) & =\frac{1}{3}\left\langle\operatorname{Tr}\left[W\left(\beta, N_{\tau}, \boldsymbol{x}\right) W^{\dagger}\left(\beta, N_{\tau}, \boldsymbol{x}+\mathbf{r}\right)\right]\right\rangle, \\
W_{S}^{\text {bare }}\left(\beta, N_{\tau}, r\right) & =\frac{1}{3}\left\langle\operatorname{Tr}\left[W\left(\beta, N_{\tau}, \boldsymbol{x}\right) S\left(\beta, N_{\tau}, \boldsymbol{x} ; \mathbf{r}\right) W^{\dagger}\left(\beta, N_{\tau}, \boldsymbol{x}+\mathbf{r}\right) S^{\dagger}(\beta, 0, \boldsymbol{x} ; \mathbf{r})\right]\right\rangle .
\end{aligned}
$$


Since the Wilson line $W$ itself is not gauge invariant, $C_{S}^{\text {bare }}$ vanishes unless a gauge-fixing procedure is used. Here, we fix to Coulomb gauge $[10,11]$. In the definition of $W_{S}^{\text {bare }}$, we use spatial Wilson lines $S$, which are path-ordered products of link matrices along the shortest paths from the point $\boldsymbol{x}$ to the point $\boldsymbol{x}+\boldsymbol{r}$ within the time slice $\tau$. We only consider paths aligned with the lattice axes, since off-axis paths of any shape would introduce additional path-dependent cusp and intersection divergences. We regulate the self-energy divergence associated with the path $S$ by smearing spatial links with the HYP smearing algorithm [12] that is restricted to the three spatial directions. Due to the spatial Wilson lines, $W_{S}$ is gauge invariant. As $C_{P}$ is a product of two independent traces of Wilson lines, it is gauge invariant and averages over all possible color combinations of the $Q \bar{Q}$ pair.

We calculate the Polyakov loop after each time unit (TU) of molecular dynamics and the correlators only with steps of ten TU. A detailed account of the ensembles is given in Ref. [7]. In order to reduce statistical noise for large $Q \bar{Q}$ separations, we divide the bare correlators by the squared bare Polyakov loop along the molecular dynamics (MD) history. We obtain statistical errors by applying the Jackknife procedure to the MD history. In infinite volume the expectation values of the ratios would correspond to connected correlators that are already renormalized and approach one for large separations. Through fits to the asymptotic behavior for different volumes, we confirm that the finite volume effects can be parameterized in terms of an asymptotic constant. After its subtraction, result for different volumes agree within errors at large separations.

The logarithms of the bare correlators can be interpreted as the negative bare $Q \bar{Q}$ free energies in temperature units. Due to the color averaging in the Polyakov loop correlator, the $Q \bar{Q}$ free energy is also called color-averaged free energy. The free energies from the singlet correlator and from the cyclic Wilson loop are considered as different definitions of a singlet free energy. We denote the various free energies obtained from connected correlators as subtracted free energies. We add the renormalized free energy of two isolated quarks, $2 F_{Q}$, to the subtracted free energies obtained from the correlators normalized by the squared Polyakov loop. These are the renormalized free energies, i.e. $F=F^{\text {sub }}+2 F_{Q}$. We renormalize the free energy of an isolated quark as

$$
F_{Q}\left(\beta, N_{\tau}\right)=F_{Q}^{\text {bare }}\left(\beta, N_{\tau}\right)+C_{Q}(\beta),
$$

where the renormalization constant can be written as

$$
C_{Q}(\beta)=\frac{b}{a(\beta)}+c+O\left(a^{2}\right)
$$

since the leading cutoff effects of the HISQ action are $O\left(\alpha_{s} a^{2}, a^{4}\right)$. Here, $b$ is a scheme-independent constant that cancels the divergence of $F_{Q}$, but $c$ as well as the higher order terms are scheme dependent. We obtain the renormalization constant $C_{Q}$ by combining two procedures as discussed in Ref. [6]. In the so-called $Q \bar{Q}$ procedure, we first obtain $C_{Q}$ from the static energy at zero temperature by fixing its value to $V_{S}\left(r_{i}\right)=V_{S}^{\text {bare }}\left(r_{i}\right)+2 C_{Q}=c_{i} / r_{i}$ with $c_{0}=0.954$ and $c_{1}=0.2065$. The Sommer scale $r_{0}$ and the scale $r_{1}$ are fixed in terms of a condition for the force,

$$
\left.r^{2} \frac{d V_{S}(r)}{d r}\right|_{r=r_{i}}=d_{i}, \quad i=0,1, \quad d_{0}=1.65, \quad d_{1}=1.0,
$$

and satisfy $r_{0}=0.4688(41) \mathrm{fm}$ and $r_{1}=0.3106 \mathrm{fm}$ in physical units. We define the lattice spacing in terms of $r_{1}$ using the results from Ref. [5]. Due to the limited availability of zero temperature lattices $(\beta \leq 7.825)$, we employ the direct renormalization scheme to extend $C_{Q}$ to higher $\beta$ following Ref. [13]. We invert Eq. (5) at fixed temperature in terms of two different combinations of $\beta$ and $N_{\tau}$, i.e. $T\left(\beta, N_{\tau}\right)=T\left(\beta^{\text {ref }}, N_{\tau}^{\text {ref }}\right)$, and infer the unknown quantity $C_{Q}(\beta)$ from the knowledge of $C_{Q}\left(\beta^{\text {ref }}\right)$, 


$$
C_{Q}(\beta)=F_{Q}\left(\beta, N_{\tau}\right)-F_{Q}^{\mathrm{bare}}\left(\beta, N_{\tau}\right)=F_{Q}\left(\beta^{\mathrm{ref}}, N_{\tau}^{\mathrm{ref}}\right)-F_{Q}^{\mathrm{bare}}\left(\beta, N_{\tau}\right)+C_{Q}\left(\beta^{\mathrm{ref}}\right) .
$$

It was shown that Eq. (8) can only be applied after cutoff effects in $F_{Q}$ are removed [6]. A detailed discussion of this procedure and tabulated values of $c_{Q}=a C_{Q}$ (Table V) are found in Ref. [6].

\section{Polyakov loop, single quark free energy and single quark entropy}

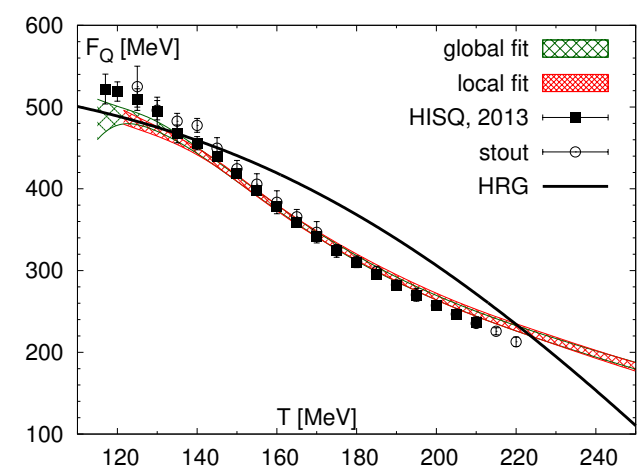

Figure 1. The free energy of a static quark. The older HISQ and and the stout results are from Refs. [14] and [4] and supersedes the former. The HRG model calculation is also from [14].

For each $N_{\tau}$ we calculate $F_{Q}^{\text {bare }}$ from the bare Polyakov loop and renormalize it as discussed. In order to extract the continuum limit, we must interpolate these results to a common set of temperatures. A detailed account of the interpolations and extrapolations is given in Ref. [6]. In Figure 1 we show the continuum limit of $F_{Q} . F_{Q}$ is quite featureless in the crossover region. Our result supersedes the older HISQ result, the differences are due to a better continuum extrapolation at low temperatures and a more precise knowledge of the renormalization constant for finer lattices (higher temperatures). We find that the hadron resonance gas (HRG) model fails to describe the Polyakov loop for $T>135 \mathrm{MeV}$. Thermal modifications of hadronic spectral functions are non-negligible well below the crossover.

We define the entropy of a single static quark through a temperature derivative of $F_{Q}$ as

$$
S_{Q}=-\frac{\partial\left(F_{Q}^{\mathrm{bare}}+C_{Q}\right)}{\partial T}=-\frac{\partial\left(F_{Q}^{\mathrm{bare}}+\frac{b}{a}\right)}{\partial T}+\frac{1}{T} \frac{\partial\left(c+O\left(a^{2}\right)\right)}{\partial \log a}=-\frac{\partial F_{Q}}{\partial T}+O\left(a^{2}\right),
$$

where the scheme dependent constant $c$ form Eq. (6) is eliminated by the derivative and the remaining scheme dependence vanishes in the continuum limit. Hence, $S_{Q}$ is scheme independent and the position of its peak defines a deconfinement temperature, $T_{S}$. We find $T_{S}=153_{-5}^{+6.5} \mathrm{MeV}$ in the continuum limit. The variation of $T_{S}$ between 150.5 and $157 \mathrm{MeV}$ for different fits defines its systematic uncertainty. In the left panel of Figure 2 we compare the continuum limit of $S_{Q}$ with older results using larger quark masses. We find that the height of the peak is much reduced in our result. We suggest the interpretation that the height of the peak is determined by the energy of the lowest-lying static-light meson, which has a contribution that is linear in the light sea quark mass. There is no linear contribution to the free energy for an unbound static quark in a chiral quark sea.

The deconfinement temperature $T_{S}$ defined in terms of $S_{Q}$ and the chiral restoration temperature $^{1} T_{\chi}$ in terms of the subtracted chiral condensate [2] agree within errors even before taking the continuum limit, as we show in Figure 2. Since this agreement is reproduced exactly in non-local

\footnotetext{
${ }^{1}$ We use the temperature $T_{\chi}$ defined on terms of the $\mathrm{O}(2)$ scaling analysis, cf. the detailed discussion in Ref. [2].
} 

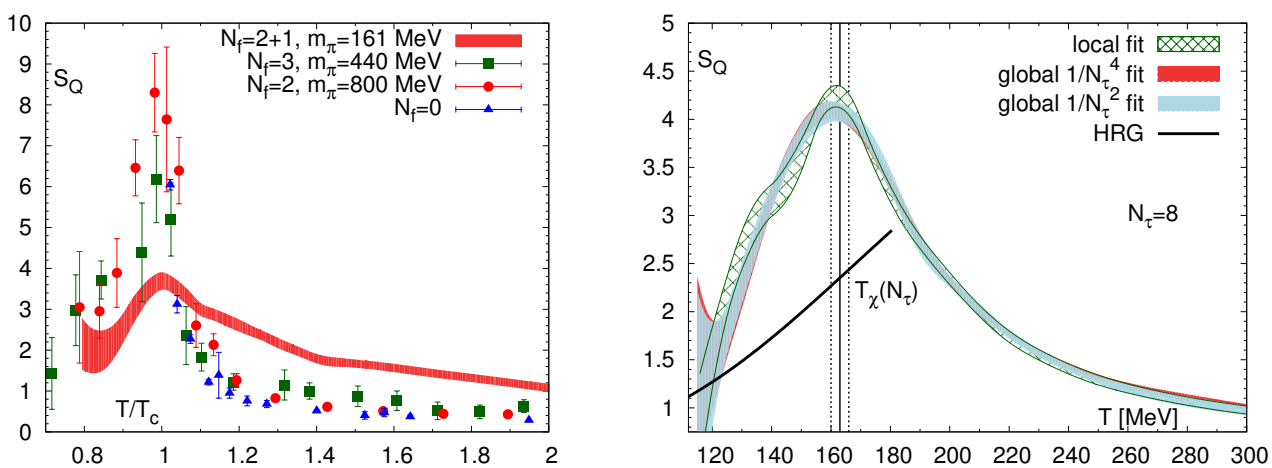

Figure 2. The entropy of a static quark. Left panel: The older results from Refs. [15] and [16] use $N_{\tau}=4$ lattices with $N_{f}=3,2$ and 0 flavors and have much larger quark masses. For each result, the temperature axis has been rescaled by the corresponding transition temperatures, i.e. $T_{S}$ for our result, and $T_{\chi}=193,200$ and $270 \mathrm{MeV}$ for the results with $N_{f}=3,2$ and 0 flavors. Right panel: The maximum $T_{S}\left(N_{\tau}\right)$ of $S_{Q}$ agrees with the chiral restoration temperature $T_{\chi}\left(N_{\tau}\right)$ [2] even at finite lattice spacing (data for $N_{\tau}=8$ ).

SU(2) PNJL model calculations [17] it appears to be a very robust feature of the QCD transition. The inflection point $T_{L}$ of the renormalized Polyakov loop cannot be defined in a scheme independent way,

$$
0=\left.\frac{1}{L} \frac{\partial^{2} L}{\partial T^{2}}\right|_{T_{L}}=\frac{F_{Q}^{2}+2\left[S_{Q}-1\right] T_{L} F_{Q}}{T_{L}}+\frac{S_{Q}^{2}-2 S_{Q}+\left.T_{L} \frac{\partial S_{Q}}{\partial T}\right|_{T_{L}}}{T_{L}^{2}},
$$

where the first term is manifestly scheme dependent due to its dependence on $F_{Q}$. Thus, $T_{L}$ generally fails to match $T_{\chi}$ on the lattice as well as in model calculations, where parameters are usually tuned to reproduce a QCD-like crossover. We suggest using the exact equality of $T_{S}$ and $T_{\chi}$ (for vanishing chemical potential) as a better constraint to improve PNJL model calculations.

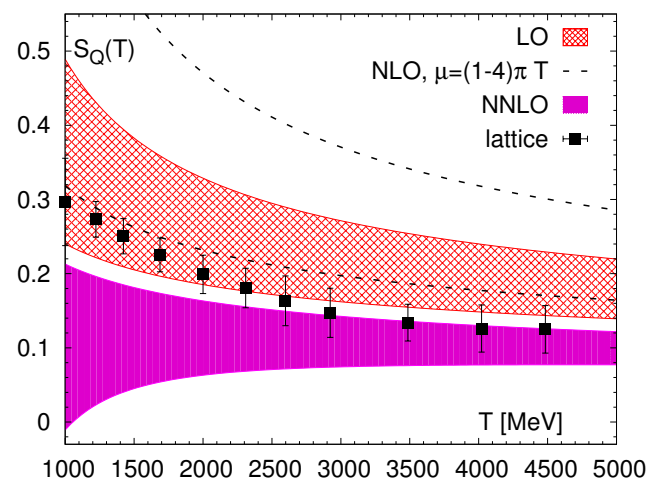

Figure 3. The entropy of a static quark at high temperature. The weak-coupling calculations are from Ref. [22].

Due to its scheme independence $S_{Q}$ is particularly well-suited for a rigorous comparison between non-perturbative results from the lattice and analytical results from weak-coupling approaches. On the lattice, we find that cutoff effects in $S_{Q}$ are of the same level as the statistical errors for very high temperatures, $T \gtrsim 1 \mathrm{GeV}$, and we can achieve a meaningful comparison even at finite lattice spacing. 
It has been known for a long time that the weak-coupling expansion does not converge as well as naïvely expected at finite temperature. The main culprit for these complications is the Debye scale, $m_{D} \sim g T$, which not only contributes odd powers to the expansion in $g$, but also is a numerically large expansion parameter ${ }^{2}$. Thus $S_{Q}$ provides a strong test of the weak-coupling expansion.

In Figure 3, we show $S_{Q}$ for $N_{\tau}=4$ together with weak-coupling results that have been obtained in Ref. [22]. The bands correspond to a variation of the scale between $\mu=\pi T$ and $\mu=4 \pi T$. For the weak-coupling result, we used $\Lambda_{\bar{M} S}=315 \mathrm{MeV}$. The lattice result lies between the leading order (LO) and the next-to-next-to-leading order (NNLO) results. Contrary to naïve intuition, the next-to-leading order (NLO) is less consistent with the lattice data than the LO. The NNLO result agrees well with the lattice result for $T>2.5 \mathrm{GeV}$. This is a considerably higher than the temperature where quark number fluctuations become compatible with weak coupling, $T>300 \mathrm{MeV}$, cf. Ref. [3] and references therein. The reason for the onset of weak-coupling behavior in $S_{Q}$ at much higher temperatures is that $S_{Q}$ is dominated by the static Matsubara mode, whereas quark number susceptibilities are dominated by the non-static Matsubara modes, cf. Ref. [22] for a detailed discussion.

\section{Polyakov loop correlator, $Q \bar{Q}$ free energy and singlet free energy}
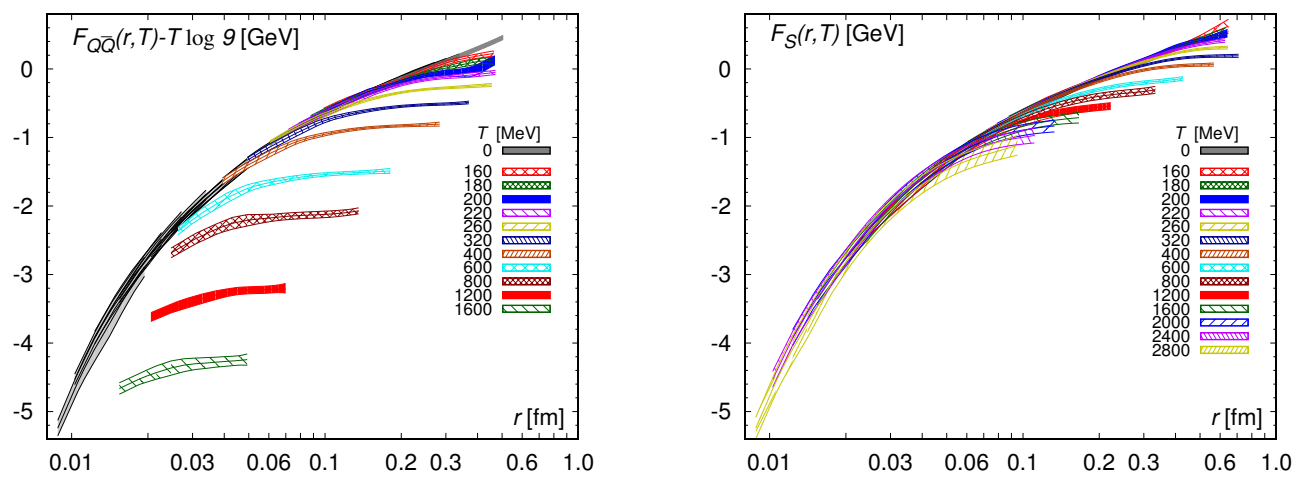

Figure 4. The color-averaged free energy (left panel) and singlet free energy (right panel) of a $Q \bar{Q}$ pair.

For each $N_{\tau}$ we calculate the subtracted $Q \bar{Q}$ free energy, $F_{Q \bar{Q}}^{\mathrm{sub}}$, from the ratio of the bare Polyakov loop correlator and the squared bare Polyakov loop and renormalize it by adding the renormalized free energy of two isolated quarks, $2 F_{Q}$. In order to extract the continuum limit, we must interpolate these results to a common set of temperatures and separations. We use the same procedure for the bare singlet correlator and extract the renormalized singlet free energy, $F_{S}$. A detailed account of the interpolations and extrapolations is given in Ref. [7].

In Figure 4 we show the continuum limit of $F_{Q \bar{Q}}$ and $F_{S}$ for a wide temperature range. The $T=0$ result in both panels represents the static energy, $V_{S}$, at zero temperature on the finest available lattice $(\beta=7.825)$. Due to asymptotic freedom the $Q \bar{Q}$ interactions for $T>0$ must be vacuum-like at small separations. Indeed, it has been shown that $F_{S}=\operatorname{Re} V_{S}+O\left(\alpha_{s}^{2} r T\right)$ in the weak-coupling limit [18]. On the lattice we find that within errors $F_{S}$ at $T>0$ is consistent with $V_{S}$ at $T=0$ for $r T<0.35$.

\footnotetext{
${ }^{2}$ For $\alpha_{s} \sim 0.4$ at $T=1 \mathrm{GeV}$ we have $g=\sqrt{4 \pi \alpha_{s}} \sim 2$. The relevant expansion parameter is then $g T / \pi T \sim 2 / 3$.
} 
We include the result for $F_{S}$ with $r T<0.3$ as a gray band in the left panel. There, we subtract a trivial factor $T \log 9$ from $F_{Q \bar{Q}}$, which is motivated by its decomposition into singlet and octet,

$$
\exp \left[-F_{Q \bar{Q}} / T\right]=\frac{1}{9} \exp \left[-F_{S} / T\right]+\frac{8}{9} \exp \left[-F_{O} / T\right]
$$

In a vacuum-like regime at small separations the color singlet is supposed to dominate and the color octet to be suppressed. For $T \lesssim 600 \mathrm{MeV}, F_{Q \bar{Q}}-T \log 9$ is consistent with $F_{S}$ and $V_{S}$ at $T=0$ for very small separations, $r T \lesssim 0.1$. At lower temperatures, i.e. $T \lesssim 220 \mathrm{MeV}$, this consistency holds even for somewhat larger separations.
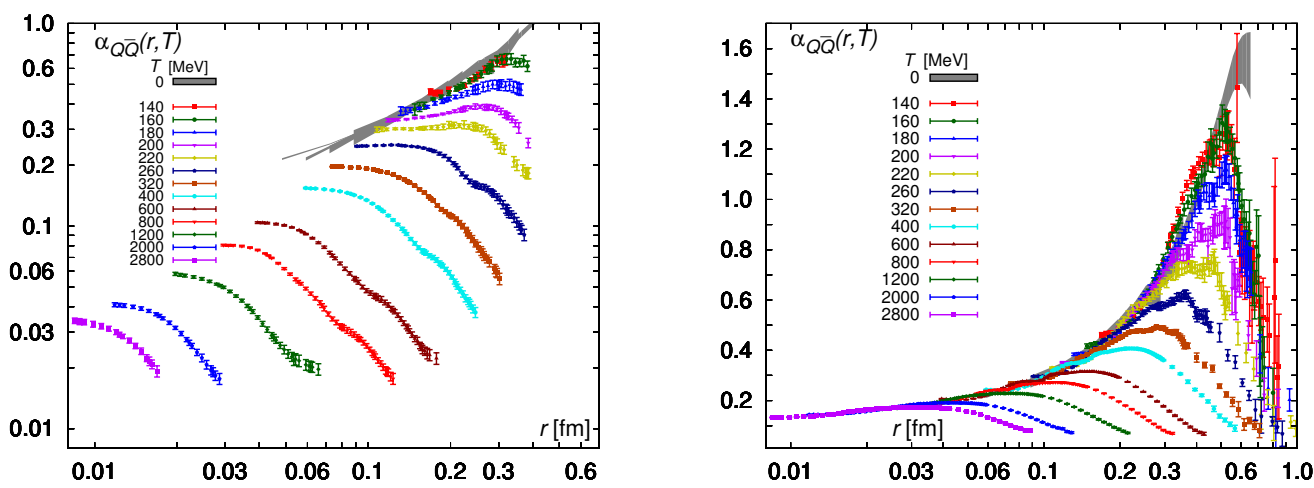

Figure 5. The effective coupling $\alpha_{Q \bar{Q}}$ in terms of the color-averaged free energy (left panel) and singlet free energy (right panel) of a $Q \bar{Q}$ pair (data for $N_{\tau}=8$ ). Note that we use a logarithmic scale in the left panel.

At larger separations the vacuum-like regime blends into a screening regime. These regimes are exposed more clearly in an effective coupling, $\alpha_{Q \bar{Q}}$, which is a proxy for the force. We define

$$
\alpha_{Q \bar{Q}}(r, \ldots)=\frac{1}{C_{F}} r^{2} \frac{\partial E(r, \ldots)}{\partial r},
$$

where $E$ may represent the static energy, the singlet free energy or the free energy. For a Coulomb potential, $\alpha_{Q \bar{Q}}$ would be constant. For a string potential, i.e. $V=a / r+b+c r$, as it is often used in phenomenology, $\alpha_{Q \bar{Q}}$ would increase quadratically.

In Figure 5 we show $\alpha_{Q \bar{Q}}$ for $F_{Q \bar{Q}}$ (left panel) and for $F_{S}$ (right panel) together with the effective coupling for $V_{S}$ indicated as $T=0$. In the right panel, we clearly see confinement at $T=0$, since $\alpha_{Q \bar{Q}}$ for $V_{S}$ increases quadratically. Though $\alpha_{Q \bar{Q}}$ for $F_{S}$ emulates this quadratic rise, it reaches a maximum after which it decays rapidly. This rapid decay indicates that correlations at separations beyond the peak at $r T \sim 0.4$ must be screened exponentially. The height of the peak, which is an indicator for the maximal strength of correlations at a given temperature, decreases from $\alpha_{Q \bar{Q}} \sim 1.2$ in the crossover region to $\alpha_{Q \bar{Q}} \sim 0.5$ at about twice the critical temperature. Hence, in this temperature range the medium can be considered as a strongly-coupled Quark-Gluon-Plasma. In the left panel, $\alpha_{Q \bar{Q}}$ defined in terms of $F_{Q \bar{Q}}$ has a positive slope only for low temperatures $T<220 \mathrm{MeV}$. For higher temperatures, however, $\alpha_{Q \bar{Q}}$ for $F_{Q \bar{Q}}$ is rather flat at small separations. The absence of a quadratic rise is due to the octet contribution. The very rapid decrease of $\alpha_{Q \bar{Q}}$ for $F_{Q \bar{Q}}$ indicates that the coloraveraged free energy is even more strongly screened than the singlet free energy. Both findings are in line with leading order predictions. 

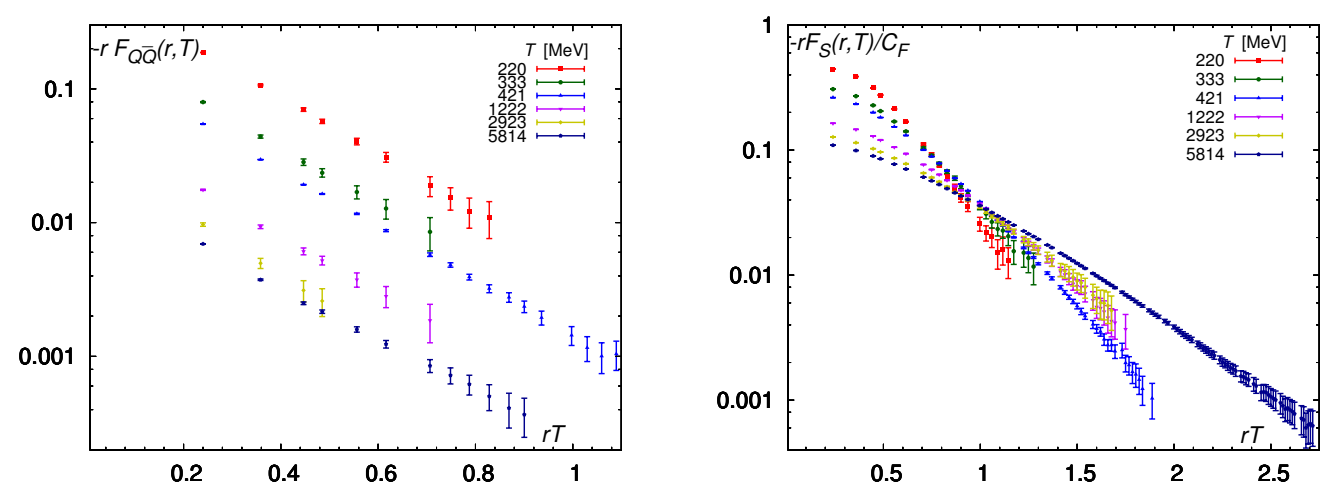

Figure 6. The screening function $S_{1}$ in terms of the color-averaged free energy (left panel) and singlet free energy (right panel) of a $Q \bar{Q}$ pair (data for $N_{\tau}=4$ ).

Color screening is studied most conveniently in terms of so-called screening functions,

$$
S_{1}=-(r T) \frac{F}{T},
$$

where $F(r)$ is a $Q \bar{Q}$ free energy. $S_{1}$ decays as $S_{1} \propto \exp [-m r]$ for exchange of a particle of mass $m$.

In Figure 6 we show the screening functions for $F_{Q \bar{Q}}$ (left panel) and $F_{S}$ (right panel). For small separations, $F_{S}$ exhibits non-screening behavior, which must be expected given its numerical similarity to $V_{S}$ at $T=0$ in this range. We see that the screening function of $F_{S}$ is not a pure exponential decay even for large separations, i.e. $r T \sim 1$, but rather that the screening mass increases for larger separations. This is not unexpected, since different weak-coupling calculations using dimensionally reduced effective field theories indicate that the asymptotic behavior is dominated by color-magnetic screening instead of color-electric screening, cf. Ref. [19] and references therein. Moreover, we find that lattices with larger volumes, i.e. aspect ratio $N_{\sigma} / N_{\tau}=6$ instead of 4 , provide a significantly better signal of the screening function for large separations, $r T>1.5$, and the asymptotic behavior is actually reached on these lattices, cf. Ref. [7]. The screening function for $F_{Q \bar{Q}}$ cannot be extracted for similarly large separations, we reach at most $r T \sim 1$ with aspect ratio $N_{\sigma} / N_{\tau}=6$. The screening mass does not show a strong variation for $r T>0.3$ and has only a fairly mild temperature dependence. A more detailed discussion of the screening masses in found in Ref. [7].

The gauge dependence of $F_{S}$ defined in terms of the Wilson line correlator raises the question, whether its screening behavior might be an artifact of a particular gauge. Gauge-dependent corrections to $F_{S}$ in general covariant gauges have been calculated at NLO in Ref. [20]. Hence, from this point of view the cyclic Wilson loop appears to be a much cleaner observable, though it has divergences due to its spatial Wilson lines. These divergences cancel in the static energy, which is defined through a ratio of a correlation functions on neighboring time slices, but persist in free energies. After regulating the divergences with link smearing, the Wilson loop can be studied at large separations without divergences though it is distorted at small separations.

In Figure 7 we show the negative logarithm of the screening function $S_{1}$ for the singlet free energy. We compare the different definitions using the Wilson line correlator in Coulomb gauge and smeared Wilson loops. Without smearing the Wilson loop decays much faster than the Wilson line correlator, but this decay is incrementally reduced through smearing. With an appropriate amount of smearing, the Wilson loop and the Wilson line correlator are numerically compatible, though the 

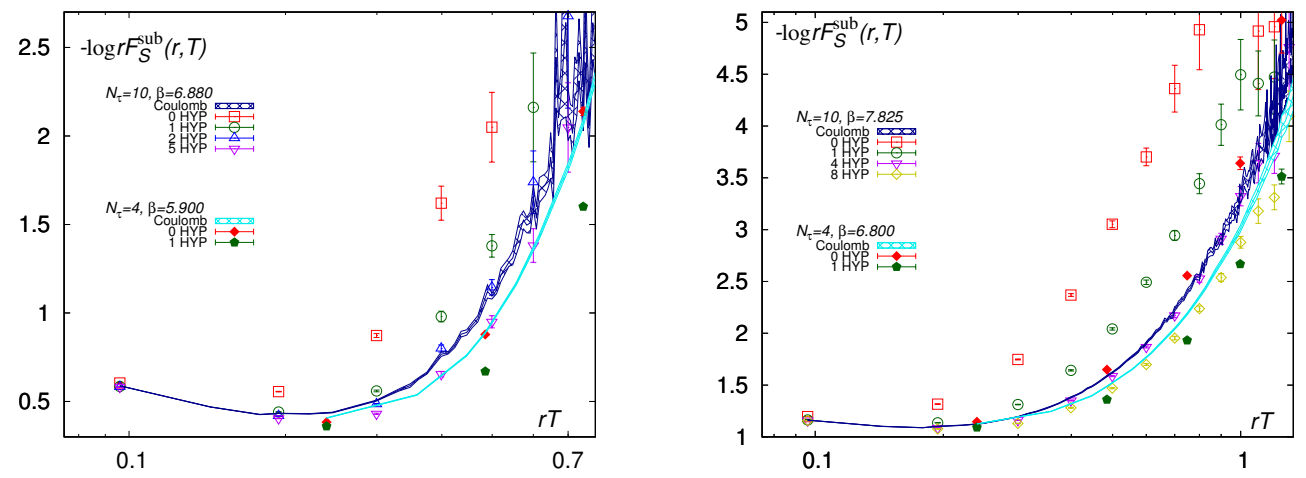

Figure 7. The negative logarithm of the screening function $S_{1}$ for the singlet free energy defined in terms of the cyclic Wilson loop and of the Wilson line correlator in Coulomb gauge for low temperatures $(T \sim 200 \mathrm{MeV}$, left panel) and high temperatures $(T \sim 480 \mathrm{MeV}$, right panel).

amount of smearing that is required depends both on the temperature and on $N_{\tau}$. We generally require more smearing for larger $N_{\tau}$ (resp. finer lattices) and for higher temperature (again finer lattices). Nevertheless, we cannot claim that we universally require the same of amount of smearing for the same lattice spacing with different $N_{\tau}$, and, due to the peculiar properties of the smearing algorithms (in integer steps), it is not entirely straightforward to vary the smearing continuously with changes of the lattice spacing. However, the gradient flow approach, which has been successfully applied for regulating the divergence of the Polyakov loop [21] and of the Polyakov loop susceptibilities[6] avoids these technical problems. Here, we can say that we reproduce the screening properties of the Wilson line correlator in Coulomb gauge with gauge-invariant smeared Wilson loops within statistical uncertainties. Thus, the screening mass of the Coulomb gauge Wilson line correlator does not seem to be bear a numerically relevant gauge dependence.

\section{Conclusions}

We have studied the Polyakov loop, the Polyakov loop correlator, a Wilson line correlator in Coulomb gauge and the cyclic Wilson loop in full QCD with $2+1$ flavors of quarks nearly at the physical point. We obtain the continuum limit of static $Q \bar{Q}$ free energies as well as of the free energy and entropy of a static quark up to high temperatures and study deconfinement and color screening.

We demonstrate that the entropy and thus the underlying Polyakov loop is a useful order parameter for deconfinement in full QCD, which allows us to establish a well-defined and scheme-independent deconfinement temperature, $T_{S}=153_{-5}^{+6.5} \mathrm{MeV}$ in the continuum limit. $T_{S}$ is numerically consistent with the temperature of chiral restoration even at finite lattice spacing. We find that lattice and weakcoupling results at NNLO for the entropy become consistent at $T>2.5 \mathrm{GeV}$.

The free energies of static $Q \bar{Q}$ pairs in the thermal medium are consistent with the zero temperature static energy for small separations as required by asymptotic freedom, the singlet free energy up to $r T \sim 0.35$ and the color- averaged free energy up to $r T \lesssim 0.1$. The color-averaged free energy is numerically consistent with the naïve decomposition into singlet and octet contributions. With an effective coupling for $F_{S}$, we highlight the remnants of the confining forces in the thermal medium as well as the onset of the screening regime. 
We study screening functions for color-averaged and singlet free energies. We analyze finite volume effects locate the asymptotic screening regime. Lastly, we show that the smeared cyclic Wilson loop and the Wilson line correlator in Coulomb gauge exhibit the same screening behavior, suggesting that the screening mass from the Wilson line correlator may be gauge independent.

\section{Acknowledgments}

We acknowledge the support by the DFG Cluster of Excellence "Origin and Structure of the Universe". The calculations have been carried out on the computing facilities of the Computational Center for Particle and Astrophysics (C2PAP) under the three Universe cluster grants "Static Quark Correlators in lattice QCD at nonzero temperature" for 2014, 2015 and 2016 (project ID pr83pu). J. H. Weber was supported by the Universe cluster for the seed project "Simulating the Hot Universe", by the Bundesministerium für Bildung und Forschung (BMBF) under grant "Verbundprojekt 05P2015 - ALICE at High Rate (BMBF-FSP 202) GEM-TPC Upgrade and Field theory based investigations of ALICE physics" under grant No. 05P15WOCA1 and by the Kompetenznetzwerk für Wissenschaftliches Höchstleistungsrechnen in Bayern (KONWIHR) for the Multicore-Software-Initiative with the project "Production of gauge configurations at zero and nonzero temperature" (KONWIHR-IV).

\section{References}

[1] L. D. McLerran and B. Svetitsky, Phys. Rev. D 24, 450 (1981).

[2] A. Bazavov et al., Phys. Rev. D 85, 054503 (2012).

[3] S. Mukherjee, P. Petreczky and S. Sharma, Phys. Rev. D 93, no. 1, 014502 (2016).

[4] S. Borsanyi et al. [Wuppertal-Budapest Collaboration], JHEP 1009, 073 (2010).

[5] A. Bazavov et al. [HotQCD Collaboration], Phys. Rev. D 90, 094503 (2014).

[6] A. Bazavov, N. Brambilla, H.-T. Ding, P. Petreczky, H.-P. Schadler, A. Vairo and J. H. Weber, Phys. Rev. D 93 (2016) no.11, 114502.

[7] A. Bazavov, N. Brambilla, P. Petreczky, A. Vairo and J. H. Weber, TUM-EFT 81/16, in preparation.

[8] http://www.physics.utah.edu/ detar/milc/milc_qcd.html

[9] http://www.usqcd.org/software.html

[10] O. Kaczmarek, F. Karsch, P. Petreczky and F. Zantow, Phys. Lett. B 543, 41 (2002).

[11] S. Digal, S. Fortunato and P. Petreczky, Phys. Rev. D 68, 034008 (2003).

[12] A. Hasenfratz and F. Knechtli, Phys. Rev. D 64, 034504 (2001).

[13] S. Gupta, K. Huebner and O. Kaczmarek, Phys. Rev. D 77, 034503 (2008).

[14] A. Bazavov and P. Petreczky, Phys. Rev. D 87, no. 9, 094505 (2013).

[15] P. Petreczky and K. Petrov, Phys. Rev. D 70, 054503 (2004).

[16] O. Kaczmarek and F. Zantow, hep-lat/0506019.

[17] J.P. Carlomagno, private communication.

[18] N. Brambilla, J. Ghiglieri, P. Petreczky and A. Vairo, Phys. Rev. D 82, 074019 (2010).

[19] P. Petreczky, J. Phys. G 39, 093002 (2012).

[20] Y. Burnier, M. Laine and M. Vepsalainen, JHEP 1001, 054 (2010) Erratum: [JHEP 1301, 180 (2013)].

[21] P. Petreczky and H.-P. Schadler, Phys. Rev. D 92, no. 9, 094517 (2015).

[22] M. Berwein, N. Brambilla, P. Petreczky and A. Vairo, Phys. Rev. D 93, no. 3, 034010 (2016). 